\title{
Design of Modular, Shape-transitioning Inlets for a Conical Hypersonic Vehicle
}

\author{
Rowan J. Gollan* \\ NASA Langley Research Center, Hampton, Virginia 23681, USA \\ Michael K. Smart ${ }^{\dagger}$ \\ University of Queensland, Brisbane, Queensland 4072, Australia
}

\begin{abstract}
For a hypersonic vehicle, propelled by scramjet engines, integration of the engines and airframe is highly desirable. Thus, the forward capture shape of the engine inlet should conform to the vehicle body shape. Furthermore, the use of modular engines places a constraint on the shape of the inlet sidewalls. Finally, one may desire a combustor crosssection shape that is different from that of the inlet. These shape constraints for the inlet can be accommodated by employing a streamline-tracing and lofting technique. This design technique was developed by Smart for inlets with a rectangular-to-elliptical shape transition. In this paper, we generalise that technique to produce inlets that conform to arbitrary shape requirements. As an example, we show the design of a body-integrated hypersonic inlet on a winged-cone vehicle, typical of what might be used in a three-stage orbital launch system. The special challenge of inlet design for this conical vehicle at an angle-of-attack is also discussed. That challenge is that the bow shock sits relatively close to the vehicle body.
\end{abstract}

\section{Nomenclature}

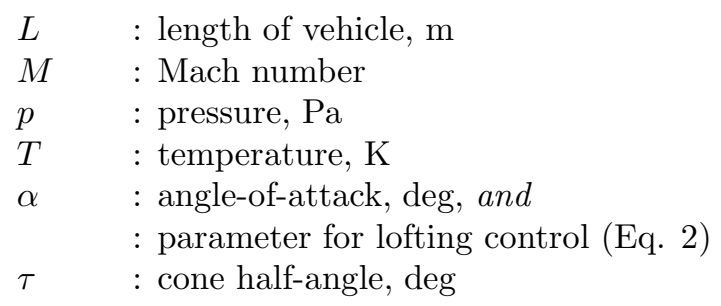

Subscripts

$\infty \quad$ : free stream conditions

$1 \quad$ : inlet entrance conditions

\section{Introduction}

For access to space, the use of an airbreathing hypersonic propulsion system in a multi-staged launch vehicle offers a much higher specific impulse than using a rocket over the hypersonic portion of the trajectory. In the design of such scramjet-propelled hypersonic vehicles, the aerodynamic integration of the airframe and the engine is a key concern. This contrasts markedly from subsonic/supersonic vehicle design, or as Heiser and Pratt ${ }^{1}$ comment, it is "a far cry from the early days of placing jet engines on pods to completely isolate them from the airplane aerodynamics." Along with integration of the scramjet engine with the airframe,

\footnotetext{
${ }^{*}$ Visiting Researcher, National Institute of Aerospace

${ }^{\dagger}$ Professor, Centre for Hypersonics
} 
there are other desirable characteristics for the engine design: good performance over a desired Mach number range; and fixed geometry to reduce structural complexity. For some design scenarios, one may require a combustor cross-section shape that differs from the inlet capture. For example, an elliptical combustor is a good compromise combustor shape based on both structural and fluid dynamic considerations. Further details about the design constraints for an airframe-integrated scramjet engine are discussed by Pinckney ${ }^{2}$ and Smart. ${ }^{3}$

In the work here, we assume that our inlet designs will be matched to an elliptical combustor. The above list of characteristics then places constraints on the hypersonic inlet: airframe-integrated at the inlet start, and an elliptical shape at the throat. In this work, we base our inlet designs on streamline-tracing techniques. Streamline-traced inlets are typically used in a modular arrangement to meet a given mass capture requirement. This further constrains the inlet capture shape, that is, the shape should allow for side-by-side placement of engine modules.

A proposed solution to these shape constraints is a three-dimensional inlet with a shape transition from a body-fitted shape to an elliptical shape at the throat. Design and testing of this class of three-dimensional shape-transitioning inlets began with the work of Hartill, ${ }^{4}$ Kiersey and Snow, ${ }^{5}$ and Kutshenreuter ${ }^{6}$ in the 1960s. However, at that time, it was difficult to predict performance for this class of inlet short of expensive and extensive wind-tunnel testing; computational tools of high enough fidelity were not available. In 1999, Smart ${ }^{3}$ renewed interest in this class of inlets with a paper which detailed a design technique for inlets with a rectangular-to-elliptical shape transition (REST). Experiments were perfomed by Smart ${ }^{7}$ on a Mach 6.0 REST inlet in 2001. The results indicated that the on-design performance of the inlet closely matched the design intent, thus serving as a verification of the design methodology.

REST inlets are ideal to integrate with a vehicle with a planar forebody. However, for other vehicle body shapes, the REST inlet is not as tightly integrated with the airframe as one would like. This leads to the purpose of this paper: we extend the design technique of Smart ${ }^{3}$ to include integration with arbitrary body shapes. Section II begins by reviewing the original design technique and then presents the new features as part of the generalisation. As an application of the new features, we report on progress towards the development of a shape-transitioning inlet for integration with a conical vehicle. Before presenting the new inlet design, Section III discusses the flow field on a proposed conical vehicle body in some detail and highlights some of the new challenges this non-uniform flow field presents for inlet design. The design methodology is applied to this new inlet configuration in Section IV. In particular, the inlet is part of a scramjet engine for use on a hypersonic vehicle for access-to-orbit purposes. This hypersonic vehicle would be the second stage (see Figure 1) of a three-stage orbital launch system as proposed by Smart and Tetlow. ${ }^{8}$
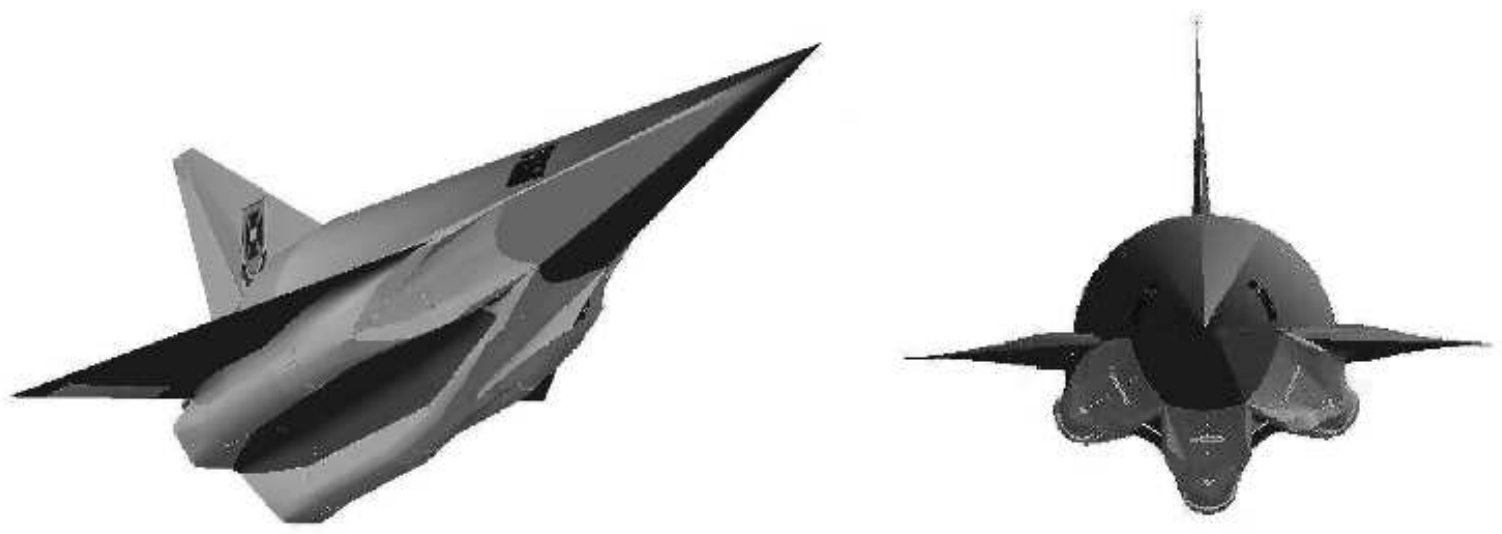

Figure 1. A winged-cone vehicle with 3 REST inlets installed. Source: Smart and Tetlow ${ }^{8}$ 


\section{Design technique}

\section{Overview}

The design method presented by Smart $^{3}$ can be separated into two parts: 1) the generation of an inviscid inlet based on streamline-tracing and lofting, and 2) the application of a viscous correction to the inviscid inlet. The first part of the design, the generation of an inviscid inlet, can be further divided into a number of steps shown below (taken from p. 410 of Smart $^{3}$ ).

1. Calculate a desirable axisymmetric compression field that has the same entrance Mach number and pressure ratio as required for the inlet.

2. Generate a stream-traced inlet using a rectangular-like capture shape such as that shown in Figure 2; this is designated as shape A.

3. Generate a second stream-traced inlet shape using a capture shape similar to shape A, but with radiused corners; this is designated as shape B (shown in Figure 2).

4. Generate a third inlet shape that has an elliptical throat of the same area as shape A at the throat; this is designated as shape $\mathrm{C}$ (shown in Figure 2).

5. Smoothly combine all three inlet shapes to form a REST inlet that has the capture shape of A, the cross-sectional shape of B at the cowl closure, and the same throat shape as C.

Tracing shape

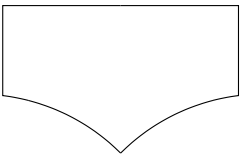

A:

B:
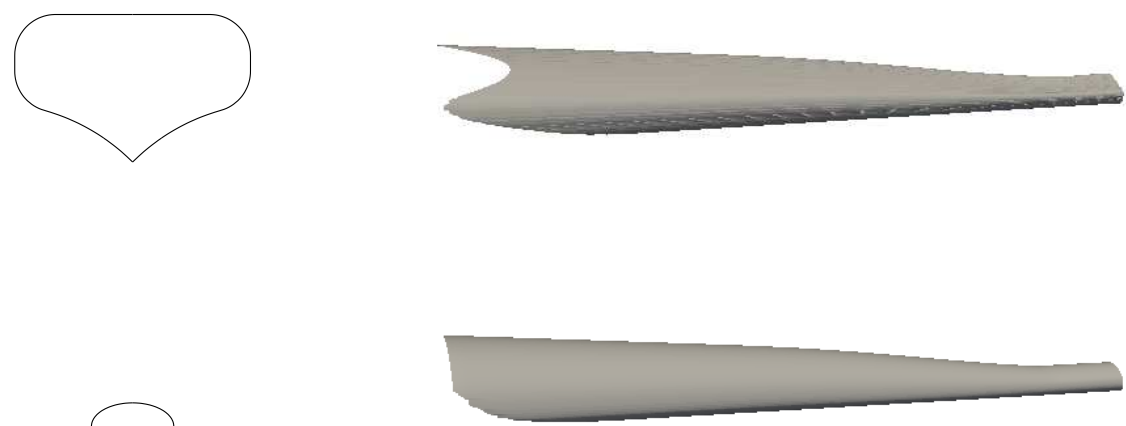

C:

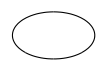

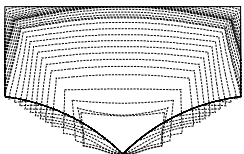
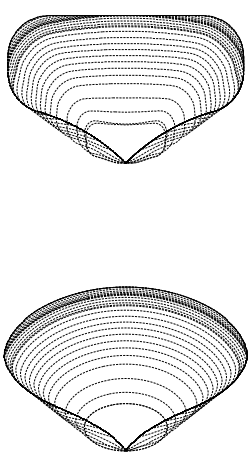

Figure 2. Inlets generated as part of streamline-tracing through the compression field. Note that the inlet based on the ellipse $\mathrm{C}$ is streamline-traced in the reverse direction, hence the small size of shape $\mathrm{C}$ compared to $\mathrm{A}$ and $\mathrm{B}$.

In the process just described, shape B is traced from the front of the axisymmetric compression field. There is a subtle problem with this approach: shape B has a smaller capture area than shape A due to the radiused corners on shape B. This in turn means that at each corresponding axial location of inlets $\mathrm{A}$ and $\mathrm{B}$, the cross-sectional area of inlet B is smaller. When inlets A and B are combined, the lofted inlet takes on the shape of inlet B at the cowl closure. Thus, at the cowl closure, the cross-sectional area is smaller than what would be expected for inlet A without lofting. Again, to repeat this subtle point, the smaller than expected area at cowl closure is a result of tracing shape B from the front of the axisymmetric compression field. 
To address this area-deficit problem, we added some flexibility to the design tool which performs the tracing and lofting. It is now possible to set a desired cross-sectional shape at the cowl closure. This is still shape $\mathrm{B}$, only now it is specified at the cowl closure instead of at the capture to the inlet. The streamlinetracing occurs in both directions, forward and reverse, from the cowl closure to form an inlet based on shape $\mathrm{B}$. The designer is free to specify the shape B at cowl closure. In practice, selecting the geometric average of the coordinates defining shapes of $\mathrm{A}$ and $\mathrm{C}$ at cowl closure works well. As a specific example of shape $\mathrm{B}$ at cowl closure, the reader may jump forward to Figure 8 which shows shape B for the inlet design presented in Section IV. Now that shape B is selected last, the order of streamline-tracing of inlets is changed: trace $\mathrm{A}$, then $\mathrm{C}$, then B.

Another addition to the design method is the generalisation to arbitrary capture shapes. With the designer free to choose the capture shape, it becomes possible to integrate inlets with a wider class of hypersonic vehicle than just planar forebodies. Section IV shows the application of the generalised capture shape feature in the design on an inlet for integration with a conical body. As part of this capture shape generalisation, the designer does not nessarily have to set the top edge of the capture shape directly. Instead, the designer can provide information about the vehicle body shape and the point of inlet attachment, and then have the tool compute the top edge of the inlet. The top edge is computed as the intersection between the shock of the compression field used for tracing and the body surface. Finally, the throat shape at $\mathrm{C}$ has been generalised to a superellipse shape. Thus, the throat shape can vary from true ellipse shapes to more rectangular shapes.

In summary, the generalistion of the design method proposed by Smart ${ }^{3}$ consists of: (1) changing where shape B is specified (now at the cowl closure); (2) allowing an arbitary capture shape for shape A; and (3) allowing for a superellipse at the throat (shape C) instead of an ellipse only. In the list above, steps 3 and 4 are reversed in the new design approach. They are shown here in their modified form for clarity:

3. Generate a second stream-traced inlet that has a superelliptical throat with the same area as shape A at the throat; this is designated as shape $\mathrm{C}$. This inlet is formed by stream-tracing in reverse, from the throat forward.

4. Based on shapes $\mathrm{A}$ and $\mathrm{C}$ at the cowl closure, select a shape at the cowl closure; this is designated as shape B. Generate a stream-traced inlet using shape B by tracing in both the forward and reverse directions.

The application of the viscous correction is discussed in more detail in a subsequent section.

\section{Generation of an axisymmetric compression field}

The generation of an axisymmetric compression field is central to the whole inlet design process; it is streamlines from this axisymmetric flow field which are used in the tracing process of building the shapetransitioning inlet. There are several features, as noted by Smart, ${ }^{3}$ that are desirable for the axisymmetric compression field: maximum total pressure recovery, a maximum strength shock train to minimise length but not so strong as to induce boundary layer separation, and minimal exit flow non-uniformity. The actual compression field is a compromise based on these desirable features. It is also useful to include a constant radius centre-body in the compression field to avoid high losses associated with shock focussing at the axis of symmetry.

Given the set of trade-offs mentioned above, there is no unique optimum choice for the axisymmetric compression field for each inlet design condition. We presently use one of two methods when designing an axisymmetric compression field: (1) a reversed expander, and (2) a truncated Busemann diffuser. In our experience, the final inlet designs based on either of these compression fields are very similar. The truncated Busemann diffuser is discussed here as it is used later in the specific design of the body-integrated inlet presented in this work (Section IV). The generation of a compression field from the reversed expander approach is discussed in the original paper by Smart. ${ }^{3}$

A Busemann diffuser may be computed for a given inlet Mach number and desired pressure ratio based on the solution to the Taylor-Maccoll equations for conical flow (see Mölder and Romeskie ${ }^{9}$ for details). For practical use of the Busemann diffuser field for inlet design, the wall needs to be truncated at some non-zero angle. We call this the truncation angle: it represents the turning angle first seen by the oncoming free stream flow. It is also our experience that the use of a centre-body reduces the strength of the cowl shock which would otherwise form at the axis of symmetry in an unmodified Busemann diffuser. To assess 
the properties of the diffuser field after truncation and the addition of a centre-body, an inviscid flow field calculation is performed. For this computation, we use the inviscid shock-fitting code SEAGULL written by Salas $^{10}$ at the NASA Langley Research Center. Some (human) iteration is required in the selection of the truncation angle and the size of centre-body radius. The iteration is stopped when the compression ratio is within $2 \%$ of the target compression ratio for the inlet design.

\section{Streamline-tracing with lofting to build inlet surface}

Once an axisymmetric compression field is obtained, the streamline-tracing process can begin. The inlet shape is generated by following steps 2, 3, 4 and 5 in the list above. In the process, three inlets (or streamtubes) are created as shown in Figure 2.. The lofted surface is built up from two lofting processes: the first part from tip to cowl closure lofts inlets A and B; and the second part from cowl closure to the the throat lofts inlets B and C. The lofting of inlets is based on the procedure proposed by Barger. ${ }^{11}$ If each cross-section is parameterised as some function of $y$, then the lofted values in the blending of inlets A and B are computed as:

$$
f(y)=\left[f_{A}(y)\right]^{1-E(x)}\left[f_{B}(y)\right]^{E(x)}
$$

where

$$
E(x)=\left(\frac{x-x_{c a}}{x_{c c}-x_{c a}}\right)^{\alpha}, \quad \alpha>0
$$

In the above, $f_{A}$ and $f_{B}$ are functions describing the cross-section properties ${ }^{\mathrm{a}}$ from inlets $\mathrm{A}$ and $\mathrm{B}$ respectively, $x_{c a}$ is the axial location of capture, $x_{c c}$ is the axial location of cowl closure and $\alpha$ is a parameter in the lofting procedure chosen by the designer. A similar expression may be written for the lofting of inlets $\mathrm{B}$ and $\mathrm{C}$, in this case going from cowl closure to the throat. Smart ${ }^{3}$ states that values of $\alpha$ between 1.0 and 5.0 tend to produce sensible shape transitions.

\section{Viscous correction to inlet surface}

The lofted inlet surface is based on an inviscid flow field. In practice, boundary layer growth will reduce the available area for the core flow. This reduction of area would lead to an effective compression ratio larger than intended. To correct for this, the surface is enlarged by considering the development of the boundary layer within the inlet. The idea is to enlarge the surface so that the core flow is similar to the inviscid flow produced in the first part of the design.

It is difficult to perform a rigorous boundary layer calculation for this complex 3D geometry: the corner flows and regions of shock-wave interaction present challenges. For design purposes, the small crossflow assumption is used when calculating boundary layer displacement thickness. As part of the process, the computed boundary layer displacement is smoothed to give the final inlet surface.

\section{Flow field considerations for a conical vehicle at angle-of-attack}

Previously, REST inlets have been designed for attachment to a planar (2D) vehicle. In the earlier work by Smart and Tetlow, ${ }^{8}$ REST scramjet engines were included on a conical vehicle design. However, this work was a system level study addressing the operability of a 3-stage access-to-space vehicle, where the second stage was scramjet propulsion. The complexities of the flow field on the vehicle underbody and its ramifications on the inlet design were not considered in detail.

Consider two vehicles, one planar and one conical, flying through the same flow conditions and presenting the same compression angle to the flow. There are two marked differences between the flow over a planar vehicle body and a conical one: (1) the shock layer on the conical vehicle experiences a 3D-relieving effect, that is, it sits closer to the vehicle body than the shock layer on the planar vehicle; and (2) the flow in the planes cutting the longitudinal axis is non-uniform, that is, flow conditions vary from the body to the shock. When the conical vehicle flies at an angle-of-attack, the flow is no longer uniform in the circumferential direction either.

\footnotetext{
${ }^{a}$ Not only do these functions give geometric properties (cross-section coordinates) to be lofted, but also the flow properties. This gives an estimate of flow conditions on the lofted inlet surface for later use in the viscous correction process.
} 
The thinner shock layer on the conical body will influence the capture shape. We may anticipate that the capture shapes for the new inlet class will be more 'squat' (have a higher width-to-height aspect ratio) compared to those inlets attached to planar bodies. In other words, the inlet shapes may require 3D-relieving in line with the relieving that 3D conical flow field undergoes. The other issue of non-uniform inflow, both in flow properties and flow angularity, may also need to be accounted for in the inlet design. In the present work, we ignore the details of this issue and just take an average of flow properties in the plane as the inflow condition for design purposes. In future work, we plan to include the non-uniform inflow as part of the inlet performance analysis.

\section{Calculating flow about the vehicle body}

Some level of care was taken with regards to calculating the flow on the vehicle body. It was important to have a good representation of the flow on the conical vehicle so that we could address the question of how the inlet design is altered for this class of hypersonic vehicle. Several approximate theories ${ }^{12-14}$ for computing the hypersonic flow over a cone at an angle-of-attack were attempted but none seemed appropriate for this vehicle configuration. With a cone half-angle of $5^{\circ}$ and a nominal angle-of-attack of $2^{\circ}$, the angle-of-attack is $40 \%$ of the half-angle or $\alpha / \tau=0.4$; not a small angle-of-attack relative to the slender vehicle. The theories are typically only valid for small angles-of-attack when $\alpha / \tau \ll 1$ or in the hypersonic limit at large Mach numbers. Instead, an inviscid CFD calculation was used to compute the flow field about a representative cone.

The simulated geometry is a circular cone with a half-angle of $5^{\circ}$ and length of $16.66 \mathrm{~m}$. The wings of the winged-cone vehicle were ignored for the purposes of estimating the flow conditions on the vehicle underbody. A $2 \mathrm{D}$ body-fitted grid was generated in the longitudinal-radial plane using transfinite interpolation, as shown in Figure 3(a), and then rotated about the axis of revolution to form a 3D volume grid (Figure 3(b)). The 2D grid was moderately clustered towards the body surface to give better resolution of the varying properties in the shock layer. The simulation exploits the symmetry of the flow field, and so the grid only wraps around half of the cone. The solution was computed on meshes with 27000 cells $(30 \times 30 \times 30)$ and 216000 cells $(60 \times 60 \times 60)$. The computed flow properties and shock location did not show any discernible differences between the two grids, and so the higher resolution mesh was considered sufficient to estimate the inlet inflow conditions. All of the results that follow were obtained using the higher resolution mesh.

The NASA Langley Research Center code VULCAN ${ }^{15}$ was used to compute the inviscid flow over the cone. The body of the vehicle was set as a slip wall boundary condition. A symmetry boundary condition was used along the symmetry planes. At the outflow plane, a first-order extrapolation boundary condition was applied. The supersonic inflow condition was applied at the remaining boundaries. The free stream conditions were $M_{\infty}=12.0, p_{\infty}=496.0 \mathrm{~Pa}$ and $T_{\infty}=240.0 \mathrm{~K}$, and set at a $2^{\circ}$ angle-of-attack. The air was modelled as a calorically perfect gas with gas constant, $R=287.1 \mathrm{~J} /(\mathrm{kg} . \mathrm{K})$, and an ideal ratio of specific heats, $\gamma=1.4$. The flow solver was used in space-marching mode, with fluxes calculated using the lowdiffusion flux-splitting scheme of Edwards. ${ }^{16}$ The flow solver typically used fewer than 100 iterations at each plane (in the marching direction) to reduce the residual by 6 orders of magnitude. ${ }^{\mathrm{b}}$ The wall clock time for these space-marching simulations was less than 2 minutes on a single core of an Intel Xeon quad-core $2.66 \mathrm{GHz}$ processor.

The computed flow field is shown in Figure 4 as contours of Mach number. The co-ordinate system used to report the results is shown in Figure 5. In Figure 6, the flow field values are plotted against the $\theta$ ordinate which is the angle made between the cone axis and a point in the flow field. So, $\theta=5^{\circ}$ corresponds to the body surface. The Mach number, static pressure and static temperature distributions are shown in Figure 6 for both the windward $\left(\phi=180^{\circ}\right)$ and leeward $\left(\phi=0^{\circ}\right)$ sides. For the design of the inlet, we are only interested in the flow conditions on the windward side. The distributions are plotted at various planes down the length of the cone $(x / L=0.25,0.5,0.75$ and 1.0) to show that the numerical solution has no length scale dependency. To give confidence that the numerical calculation was a reasonable estimate of the flow conditions, the 'exact' Taylor-Maccoll solutions for a cone with a half-angle of $5^{\circ}$ at zero angle-of-attack is also shown in Figure 6. As expected, the windward and leeward distributions for a cone at an angle-of-attack fall on either side of the values for the zero angle-of-attack cone with $5^{\circ}$ half-angle.

A set of inflow conditions are required for the inlet design. Note that the inlet inflow has already been processed once by the shock generated on the vehicle body. The results from the simulation have been

\footnotetext{
${ }^{\mathrm{b}}$ The initial few planes required far more than 100 iterations to converge, but this was expected in the first few planes where the geometry comes to a sharp point.
} 


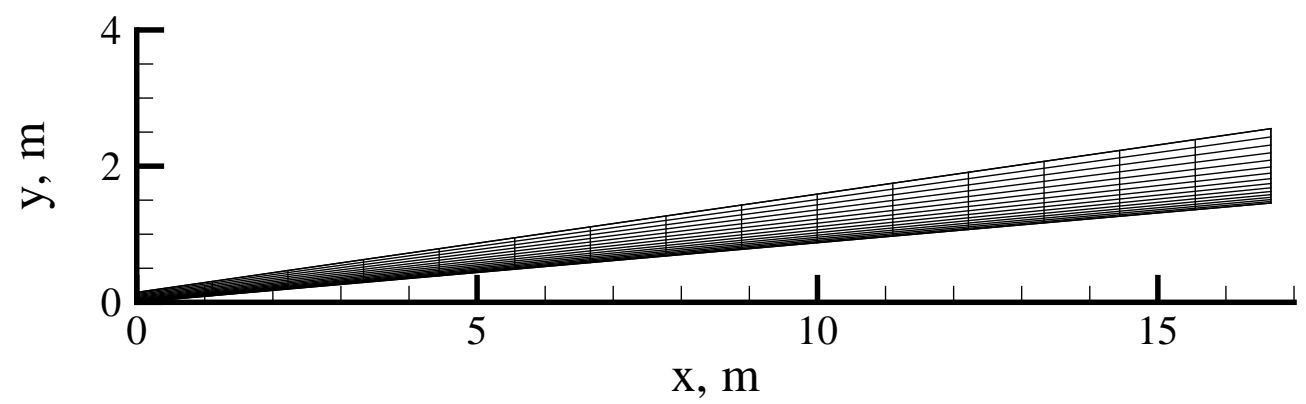

(a) 2D structured grid in XY-plane

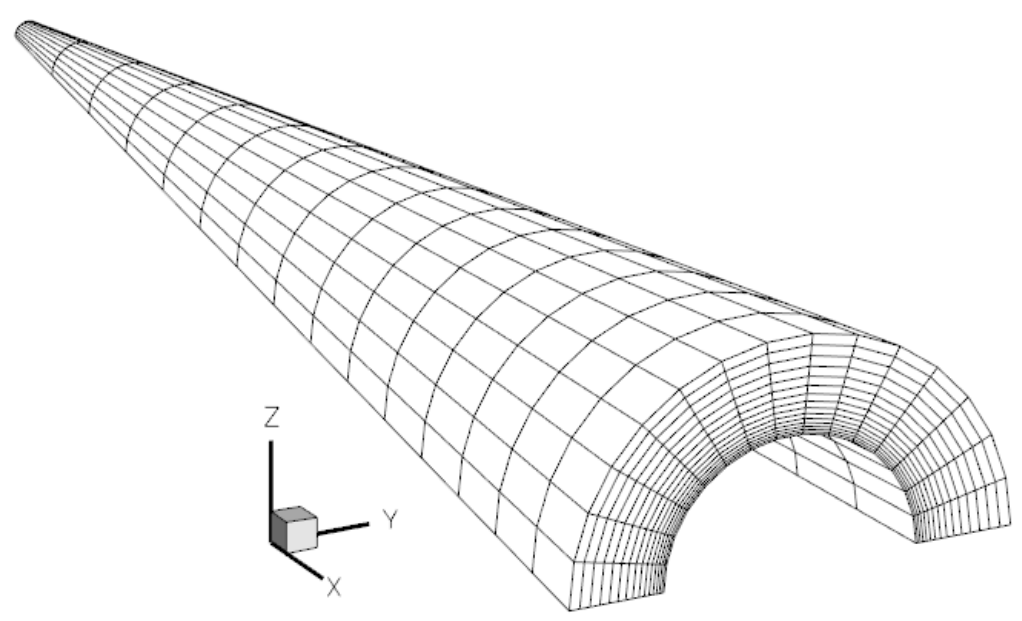

(b) $3 \mathrm{D}$ volume grid

Figure 3. Views of mesh for simulation of hypersonic flow over a $5^{\circ}$ half-angle cone. For clarity, only every fourth gridline is shown. 


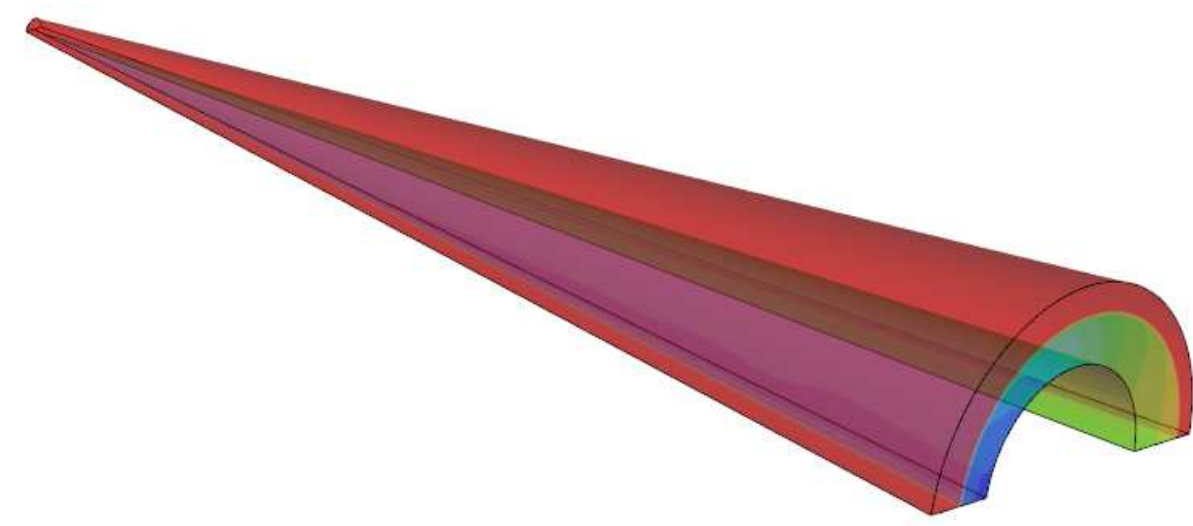

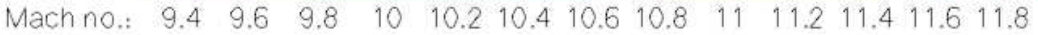

Figure 4. Contours of Mach number for a $5^{\circ}$ half-angle cone at a $2^{\circ}$ angle-of-attack in Mach 12 flow

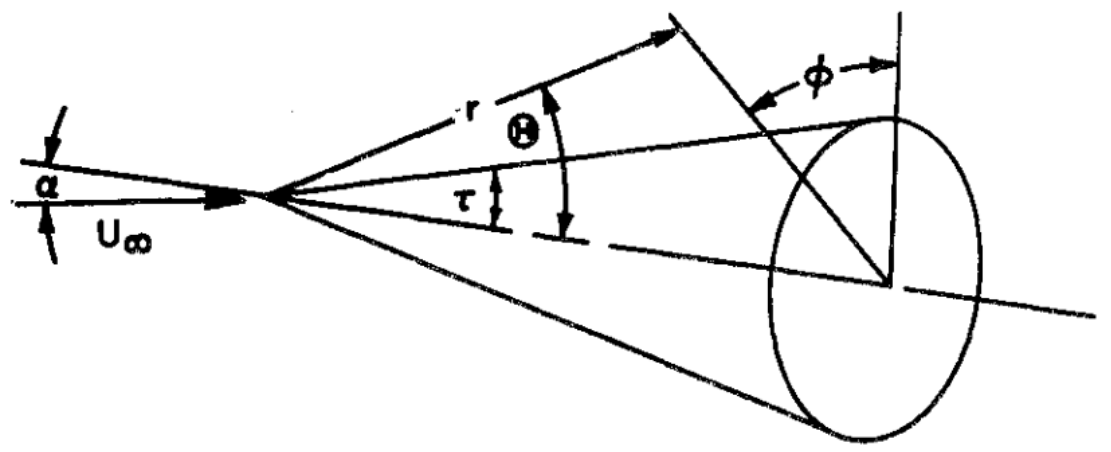

Figure 5. Coordinate system for reporting of conical flow field results. Source: Doty and Rasmussen ${ }^{13}$ 

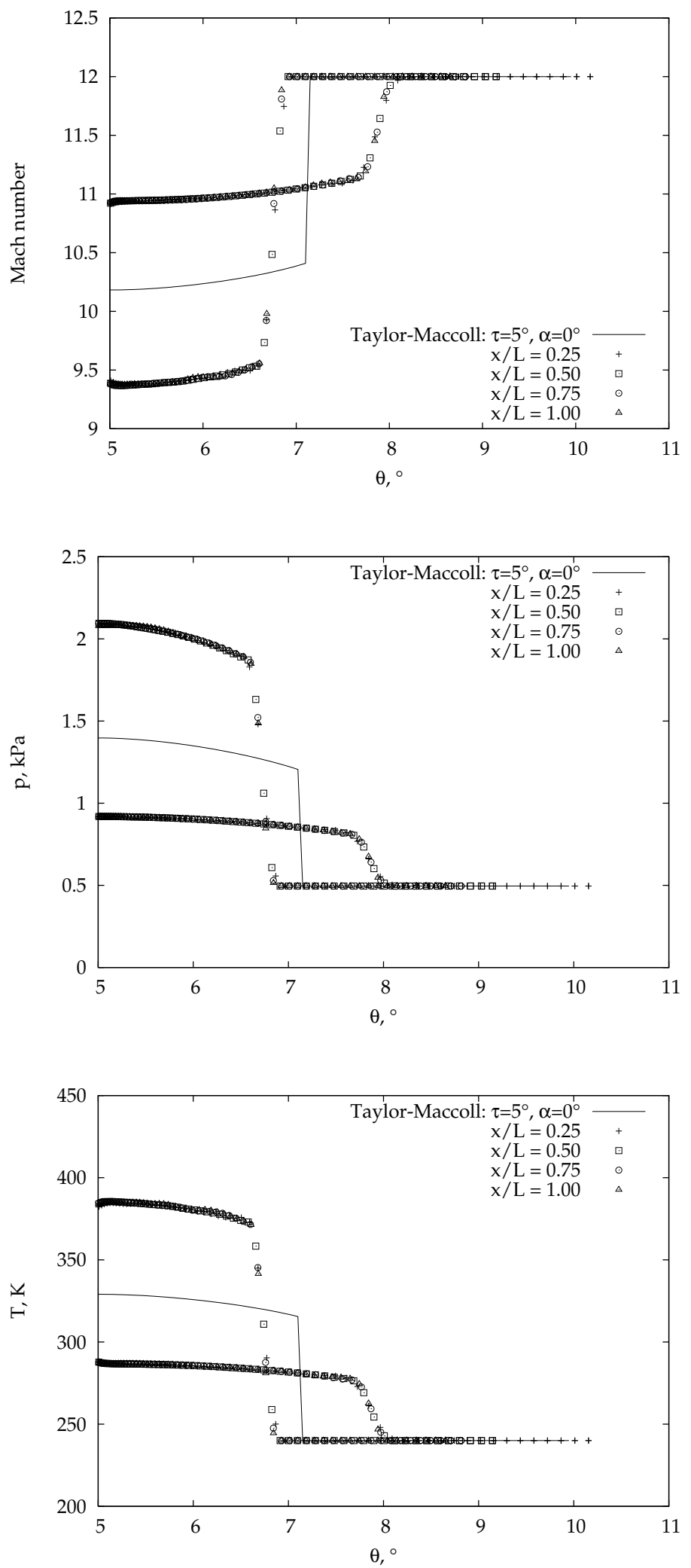

Figure 6. Distribution of flow field values between the body and shock for a $5^{\circ}$ half-angle cone at $2^{\circ}$ angle-of-attack in a Mach 12 flow. For comparison, the Taylor-Maccoll solution for a cone at zero angle-of-attack at the same flow conditions is also shown. 
area-averaged on the underside of the vehicle body to give an estimate of the inlet inflow conditions. These averaged inflow conditions are $M_{1}=9.88, p_{1}=1643 \mathrm{~Pa}$ and $T_{1}=352 \mathrm{~K}$.

\section{Inlet location and size}

Body-integrated, shape-transitioning inlets use a large amount of isentropic compression to achieve high overall efficiency. The use of isentropic compression tends to make for a longer inlet compared to conventional $2 \mathrm{D}$ inlet designs. As such, shape-transitioning inlets are located in a more forward position on the vehicle when compared to the placement of traditional engine designs. The rule-of-thumb is to place the shapetransitioning engine as far forward as possible, while ensuring that the inlet capture sits within the envelope of the vehicle shock layer. It is highly undesirable for the inlet to 'protrude' from the vehicle shock layer as this would lead to problems such as excessive cowl drag and excessive aerodynamic heating on the protruding parts.

As mentioned earlier, the 3D-relieving effect that occurs on a conical vehicle causes the bow shock to sit closer to the body and, thereby, influences the inlet capture shape. Figure 7 shows the shock location on a conical body at the nominal $2^{\circ}$ angle-of-attack. For comparison, the shock location for a $5^{\circ}$ wedge at a $2^{\circ}$ angle-of-attack is also shown in Figure 7 . This graphically demonstrates how much the thickness of the shock envelope is reduced and the follow-on effect on the design of the inlet capture shape.

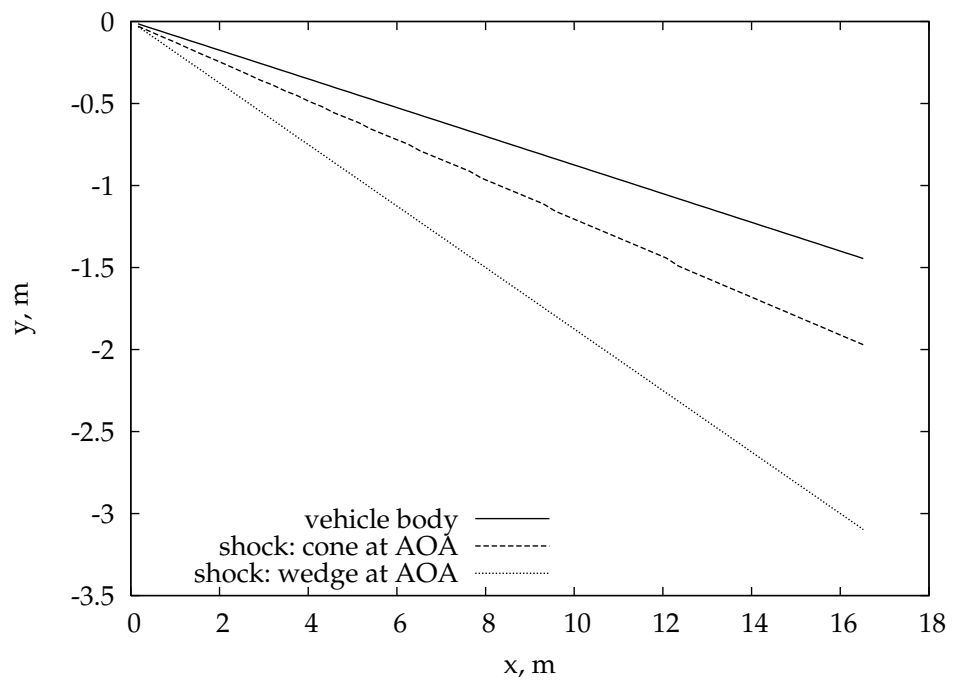

Figure 7. Shock envelope on a $5^{\circ}$ half-angle cone at $2^{\circ}$ angle-of-attack in Mach 12 flow. For comparison, the shock angle for a $5^{\circ}$ wedge at $2^{\circ}$ angle-of-attack is also shown.

The correct sizing and location of a body-integrated inlet on the vehicle body is an iterative process. To start the iterative process, the attachment location is chosen relatively close to the front of the vehicle. The attachment location refers to the point where the tip of the inlet attaches to the vehicle body. As a starting guess for this inlet design, the attachment location is chosen as $x / L=0.25$. The design will proceed so that the inlet is sized to attach at this location and meet the requirement that the entire inlet capture sits inside the shock layer at the design condition. If, after complete engine analysis, this inlet sizing (along with combustor and exhaust nozzle) does not produce the appropriate thrust, the inlet sizing may be scaled and the attachment location adjusted in accordance with the new size. For scalings between about $0.75-1.25$ of the original size, it would not be necessary to repeat the streamline-tracing design process. For scalings outside of that range, the design process should be restarted. In this manner, one would iterate towards a correct sizing and attachment location for the inlet.

\section{Inlet design}

The inlet design method was described earlier in Section II, but the new aspects of the design process are repeated here to show how they apply to this particular design example. First, the design tools have been generalised to allow for an arbitrary capture shape, thus allowing for integration with any vehicle body. The 
inlet design presented in this paper makes use of the new tool feature to streamline-trace through a userdefined capture shape. The details of the capture shape for integration on the conical body are presented below. Second, the intermediate shape for use in lofting is now specified at the point of cowl closure, and a streamtube is formed by tracing both forwards and backwards through the flow field from the point of cowl closure. An example of this intermediate shape, specified at cowl closure, is shown in Figure 8.

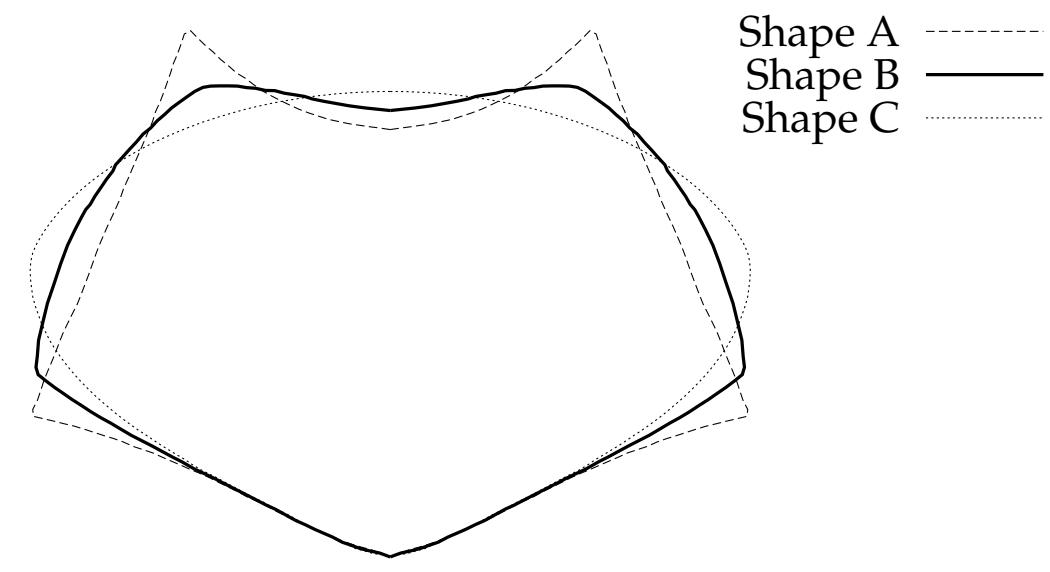

Figure 8. Shape B specified at cowl closure. Any shape may be specified; this particular example is the average of shapes $\mathrm{A}$ and $\mathrm{C}$ at the cowl closure.

\section{Choosing the number of scramjet modules}

In the paper by Smart and Tetlow, ${ }^{8}$ it was proposed to attach three modular scramjet engines to the wingedcone vehicle. In the spirit of working from their proposed design, an initial attempt was made to design an inlet which would be part of three modular scramjet engines stacked side-by-side. A frontal view of the proposed arrangement is shown in Figure 9. Note that the capture shapes are somewhat squat (large width-to-height aspect ratio), and so some difficulties were encountered with this design shape. First, the large aspect ratio challenged the robustness of the design tool: it was not always able to generate sensible shapes, or the application of the viscous correction was not well-behaved. Second, the capture shapes that did produce reasonable inlets would end with a throat that was located significantly lower than the cowl closure. This was a consequence of the need to choose squat capture shapes to streamline-trace through the axisymmetric compression field. These lowered throats are a problem as they add to total vehicle drag.

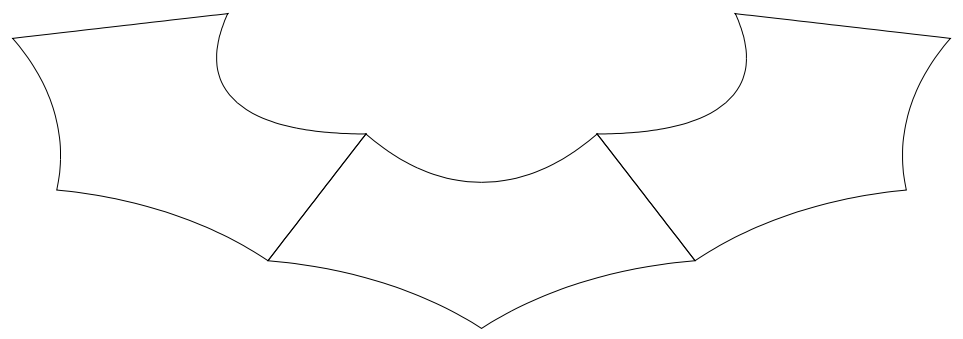

Figure 9. View of inlet capture shapes arranged as three modules on vehicle underbody. Note the top edge is not circular; it follows the vehicle body curve backwards as the the vehicle body increases in radius.

As a remedy to these problems, a fourth scramjet module can be added on the vehicle underbody as shown in Figure 10. The use of four scramjet modules leads to inlets that are less squat compared to the case when three modules are used. Thus, the tool produces somewhat more sensible design shapes. The question arises as to what penalty (or indeed, benefit) is incurred by using four scramjet engines instead of 
three. This a question that is best assessed at a system level design optimisation and we do not address that issue here. It should be possible to produce an inlet appropriate for a three-module arrangement. The key will be in producing a good compression field from which to streamline-trace the inlets. In future work, we intend to investigate the use of different compression fields when designing inlets for attachment to a conical vehicle.

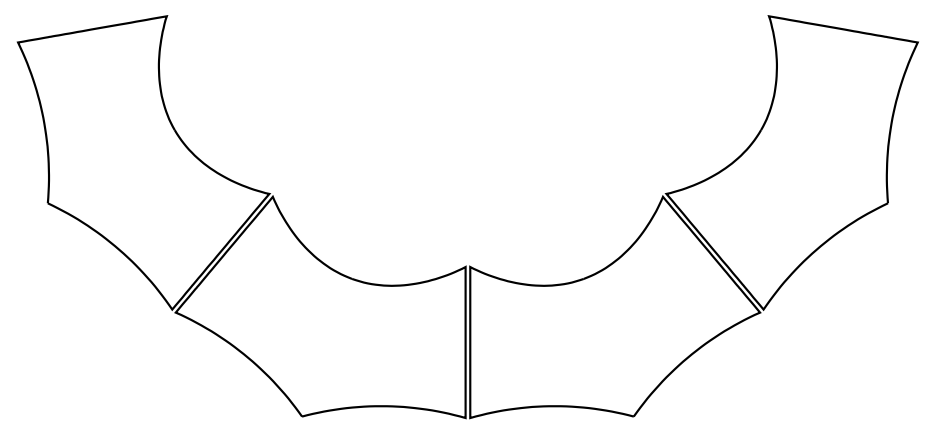

Figure 10. View of inlet capture shapes arranged as four modules on vehicle underbody.

\section{Inlet capture shape}

Utilising the new feature of arbitary capture shapes, a user-defined, customised shape was specified that allows integration to a conical vehicle. This particular customised capture shape is show in Figure 11.

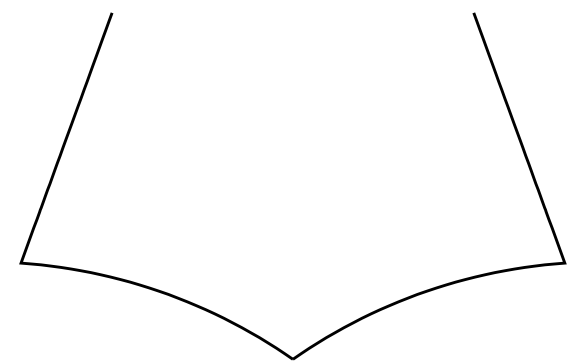

Figure 11. Example capture shape for a body-integrated inlet on a conical vehicle. Note this is the actual shape used in the present design example.

The top edge of the capture shape is not directly specified for this conical design. Instead, the designer supplies the cone half-angle. Based on this angle, the rate at which the attachment radius grows is determined. The tool then calculates where the shock of the axisymmetric compression field intersects with the vehicle body, working from the most forward point of inlet attachment to the centre (the symmetry plane). This intersection curve forms the top edge of the inlet and conforms to the vehicle body shape. This process of computing the intersection between the body and the designer's compression field is one of the new features of the design method. It also allows the design of capture shapes for integration with more general body shapes that may be of interest in the future. 


\section{Inlet geometry}

In this section, we present the preliminary inlet design. At this stage, the inlet surface has not been corrected for viscous effects, and the inlet has not been analysed with CFD. The viscous correction tool requires updating to accommodate the new shapes allowed by the generalised capture shape feature. Similarly, the process to generate structured grids for these $3 \mathrm{D}$ geometries needs to be modified for the new inlet shapes. First, the axisymmetric compression field is described. The various streamtubes which are lofted to form the inlet shape were all streamline-traced from a common compression field. A truncated Busemann diffuser was used as the axisymmetric compression field. The Busemann diffuser is characterised by the pre-shock Mach number after isentropic compression, and the turning angle at the shock: in this case, $M_{2}=6.2$ and $\beta=12.25^{\circ}$. The contour of the diffuser may be calculated based on this information using the procedure described by Mölder and Romeskie. ${ }^{9}$ To form the axisymmetric compression field, the Busemann diffuser was truncated where the diffuser contour was $4.5^{\circ}$ and a centre-body with a (non-dimensional) radius of 0.1 was added. The opening of the truncated diffuser is 1.0 in non-dimensional units. The inviscid flow through the truncated Busemann diffuser was computed with SEAGULL, ${ }^{10}$ the results of which are shown in Figure 12. A true Busemann diffuser perfectly cancels the shock at the throat of the diffuser. In the truncated diffuser, the shock is not perfectly cancelled because the non-zero initial turning angle changes the shock position.

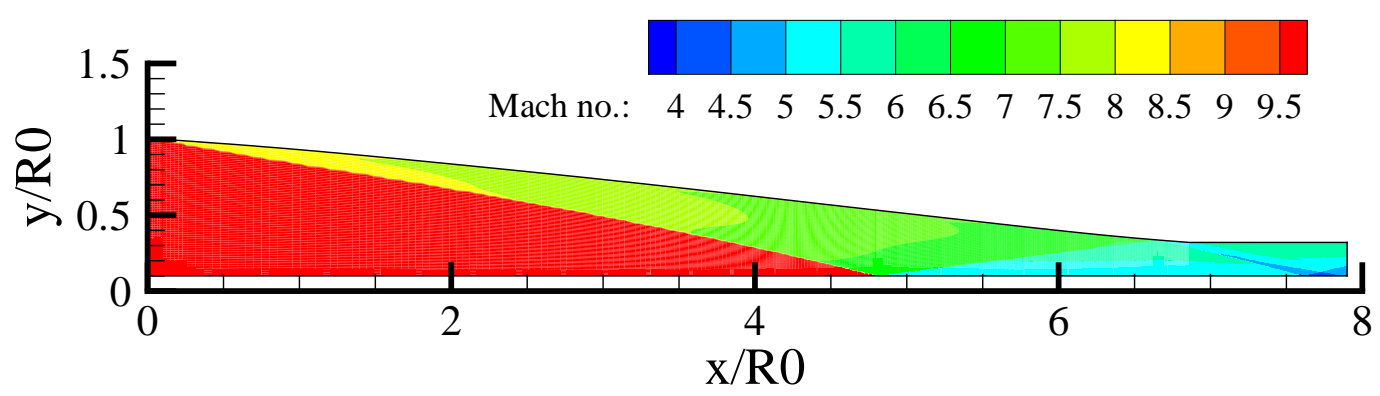

Figure 12. Contours of Mach number in the axisymmetric compression field used for streamline-tracing of the inlet

Following the method described in Section II, various streamtubes were traced through the axisymmetric compression field and lofted to form an inlet shape. The inlet shape is shown in Figure 13 in various views. At this inviscid stage of the design, this inlet has a total geometric contraction ratio of 4.3 and an internal contraction ratio of 1.5. The throat was specified as an ellipse with an aspect ratio of 2.0.

\section{Future work}

To complete this inlet design, the immediate future work involves application of the viscous correction and then an analysis of the inlet performance using viscous CFD. Part of our continuing work is to update the viscous correction tool in line with the new generalised capture shapes that our design tool now accommodates. We are also in the process of generalising our capability to rapidly build grids for these shape-transitioning inlets; the rapid grid generation is the present stumbling block towards using high-fidelity CFD at the design stage.

The issue of non-uniform inflow conditions has not been addressed at present. As a start, we plan to perform an anlysis of the inlet with a full Navier-Stokes calculation including the non-uniform inflow conditions. There is a question as to whether using averaged inflow conditions is satisfactory for design purposes or if a more complex accounting of the non-uniformities at the design stage is required. The results of the inlet flow field analysis will be used to guide the research direction on the question of non-uniform inflow.

During this work, some items of interest for future work arose. One of these items is to investigate the use of different compression fields for the streamline-tracing of the inlet which may help to form sensible shapes for the squat inlets characteristic of a three-module system. The design method is not limited to axisymmetric compression fields. However, no other types of compression field have been used because these 
(a) side view

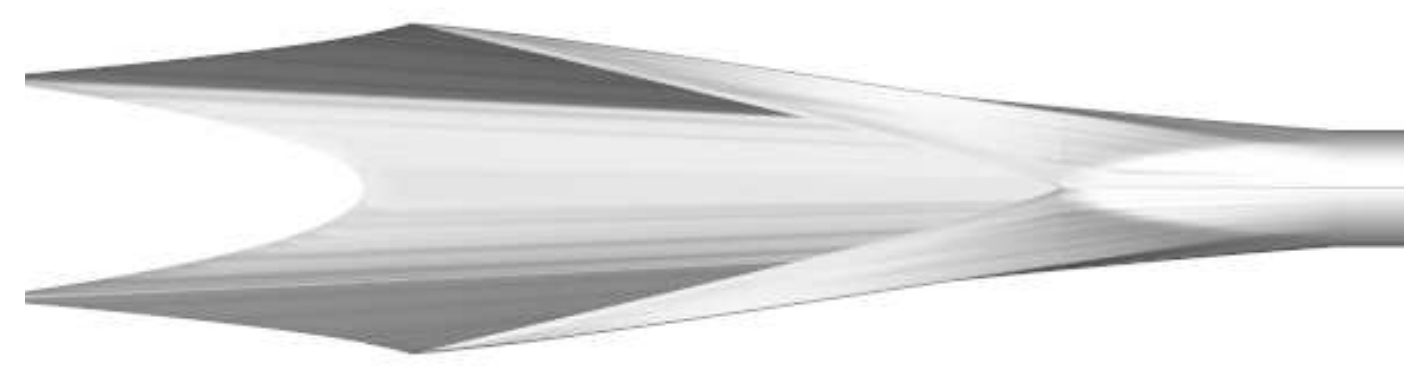

(b) bottom view

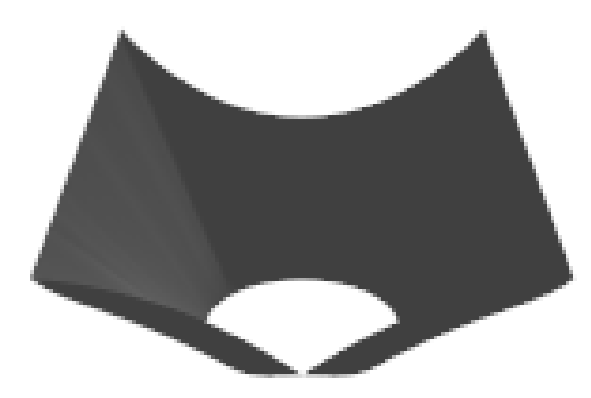

(c) front view

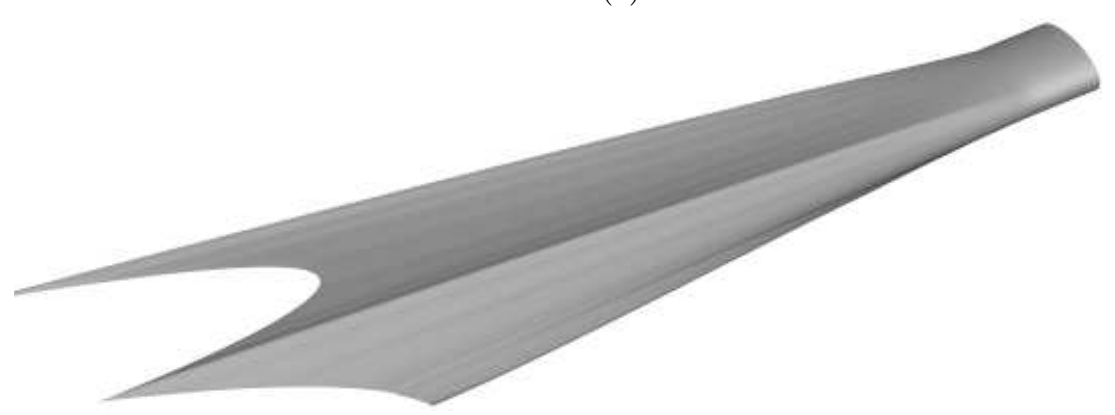

(d) view of leading edges

Figure 13. Various views of the inlet shape for integration with a conical vehicle 
axisymmetric fields have worked well for REST inlet shapes in previous designs. It would be interesting to try different compression fields when designing the body-integrated inlets for non-planar vehicle shapes.

Another item of interest was the question of the number of scramjet modules to use on this class of conical vehicle. In this instance, four modules were chosen based puerly on inlet design considerations; the larger system level design was not considered. A complementary project is currently underway to develop software tools that allow the use of high-fidelity CFD analysis in the design phase. This new capability could be applied to the configurations presented here to address system level design questions such as the optimum number of scramjet modules to use on a vehicle of this class.

\section{Concluding remarks}

This paper reported on a generalisation of the REST inlet design technique to allow for inlet integration with any arbitrary shape of realistic hypersonic vehicle configurations. Specifically, the generalisations of the design technique were: (1) the allowance for arbitrary capture shapes for integration with a wider class of hypersonic vehicle bodies, rather than just planar bodies; (2) the provision to set a superellipse at the throat, which was previously limited to an ellipse only; and (3) added flexibility in the streamline-tracing process so that an intermediate shape may be set at the cowl closure - this is to help with an area-deficit problem that arose from tracing the intermediary inlet (B) from the front of the compression field. As a demonstration of the new technique, a preliminary inlet design for attachment to a winged-cone vehicle was presented.

During the development of the new inlet, the challenges of designing for a conical vehicle at angle-ofattack were identified. As a second focus of the paper, the challenge of accommodating an inlet within the relatively thin, 3D-relieved shock layer as compared to a planar vehicle was discussed. In order to correctly size and locate the inlets on the conical body, it was found that the inlet capture shapes became quite squat.

\section{References}

\footnotetext{
${ }^{1}$ Heiser, W. H. and Pratt, D. T., Hypersonic Airbreathing Propulsion, Education Series, AIAA, Washington, DC, 1994.

${ }^{2}$ Pinckney, S. Z., "Rectangular capture area to circular combustor," TM 78657, NASA Langley Research Center, Hampton, Virginia, 1978.

${ }^{3}$ Smart, M. K., "Design of three-dimensional hypersonic inlets with rectangular-to-elliptical shape transition," Journal of Propulsion and Power, Vol. 15, No. 3, 1999, pp. 408-416.

${ }^{4}$ Hartill, W. B., "Analytical and Experimental Investigation of a Scramjet Inlet of Quadriform Shape," TR AFAPL-TR65-74, U.S. Air Force, Marquardt Corp., Aug. 1965.

${ }^{5}$ Kiersey, J. L. and Snow, M. L., "Modular Inlet Investigation," Quarterly Report AQR/66-1, Aeronautics Div., Research and Development, Applied Physics Lab., Johns Hopkins University, Baltimore, MD, Jan. 1966.

${ }^{6}$ Kutshenreuter, P. H., "Hypersonic Inlet Tests in Helium and Air," Conference paper, AIAA Propulsion Joint Specialist Conference, U.S. Air Force Academy, CO, June 1965.

${ }^{7}$ Smart, M. K., "Experimental testing of a hypersonic inlet with rectangular-to-elliptical shape transition," Journal of Propulsion and Power, Vol. 17, No. 2, 2001, pp. 276-283.

${ }^{8}$ Smart, M. K. and Tetlow, M. R., "Orbital delivery of small payloads using hypersonic airbreathing propulsion," Journal of Spacecraft and Rockets, Vol. 46, No. 1, 2009, pp. 117-125.

${ }^{9}$ Mölder, S. and Romeskie, J. M., "Modular Hypersonic Inlets with Conical Flow," AGARD CP-30, McGill University, 1968.

${ }^{10}$ Salas, M. D., "Shock fitted method for complicated two-dimensional supersonic flows," AIAA Journal, Vol. 14, No. 5, 1976, pp. 583-588.

${ }^{11}$ Barger, R. L., "A Procedure for Designing Forebodies with Constraints on Cross-Section Shape and Axial Area Distribution," TP 1881, NASA Langley Research Center, 1981.

${ }^{12}$ Cheng, H. K., "Hypersonic flows past a yawed circular cone and other pointed bodies," Journal of Fluid Mechanics, Vol. 12, 1962, pp. 169-191.

${ }^{13}$ Doty, R. T. and Rasmussen, M. L., "Approximation for hypersonic flow past an inclined cone," AIAA Journal, Vol. 11, No. 9, 1973, pp. 1310-1315.

${ }^{14}$ Merlen, A. and Andriamanalina, D., "Analytical solutions for hypersonic flow past slender power-law bodies at small angle of attack," AIAA Journal, Vol. 30, No. 11, 1992, pp. 2683-2693.

${ }^{15}$ White, J. A. and Morrison, J. H., "A Pseudo-temporal Multi-grid Relaxation Scheme for Solving the Parabolized NavierStokes Equations," AIAA Paper 99-3360, 1999.

${ }^{16}$ Edwards, J. R., "A low-diffusion flux-splitting scheme for Navier-Stokes calculations," Computers and Fluids, Vol. 26, No. 6, 1997, pp. 635-659.
} 